\title{
Low Phase Noise 2 GHz HBT Push-Push VCO Based on an Advanced Low Frequency Noise Model
}

\author{
A. SION* - P. CORTESE* - C. GOURDON* - M. CAMIADE* - J.C. NALLATAMBY** \\ M. PRIGENT ${ }^{* *}-$ J. OBREGON ${ }^{* *}$ \\ "UMS S.A.S., RD 128, BP 46, 91401 ORSAY CEDEX, FRANCE \\ ${ }^{* *}$ IRCOM - CNRS , 7 rue Jules Valles, 19100 BRIVE, France \\ tel : + 0033169330627 Fax :0033169330552 \\ E-mail : arnaud.sion@ums-gaas.com
}

\begin{abstract}
After investigation of the possible HBT low frequency noise sources and their localization in order to extract a HBT low frequency noise model, the realisation and performances of a $S$ band Voltage Controlled Oscillator (VCO) is reported. This VCO is fully integrated in MMIC technology, and is based on a push-push topology. The circuit is covering $280 \mathrm{MHz}$ tuning range around a centre frequency of $2 \mathrm{GHz}$ with a phase noise of $-95 \mathrm{dBc} / \mathrm{Hz}$ at 10 KHz offset.
\end{abstract}

\section{INTRODUCTION}

Design of low phase noise oscillators has always been challenging for microwave engineers. They are now likely to estimate the oscillator phase noise thanks to harmonic balance software, which plans the frequency fluctuations caused by low frequency noise sources. However, an accurate characterization of their low frequency noise sources is essential.

The main objective of this work was to validate a new low frequency noise model with a low phase noise $\mathrm{S}$ band push push oscillator realization.

This paper consists of two main parts :the low frequency noise model extraction, and then the VCO design and its measurement results.

\section{ADVANCED LOW FREQUENCY NOISE MODEL EXTRACTION}

\section{A. AN IMPROVED LOW FREQUENCY NOISE MODEL $O F H B T$}

1.Low frequency non-linear HBT model.

Fig. 1 shows the small signal T-model of the HBT extracted from a non-linear large signal, valid at low frequencies where the reactive element influences can be neglected.

The T-model must be preferred to the $\pi$-model in order to accurately locate the low frequency noise sources, because it exactly represents the current paths within the actual device; for example the $\mathrm{I}_{\mathrm{BE}}$ emitter current path is correctly represented, while the $\pi$-model only shows the $\mathrm{I}_{\mathrm{B}}$ current.
$\alpha_{\mathrm{F}} \mathrm{I}_{\mathrm{BEint}}$ is the current controlled current source of the transistor: it represents the part of the current generated into the base-emitter junction, injected in the basecollector junction.

2. Possible low frequency noise sources and their localization.

Microscopic physical noise sources lead to random fluctuations of conductive currents. Then, it follows that in a macroscopic electrical representation, every equivalent Norton noise current source must be associated to the conductive element where it originates. Note that all the noise sources shown in the figure are uncorrelated.

$\delta \mathrm{I}_{\mathrm{BE}}$ represents the GR and 1/f noise sources generated into the base-emitter junction

$\delta \mathrm{I}_{\mathrm{RBE}}$ represents the GR and $1 / \mathrm{f}$ noise sources of the recombination current principally present at the periphery of the base-emitter junction.

$\delta \mathrm{I}_{\mathrm{RB}}$ and $\delta \mathrm{I}_{\mathrm{RE}}$ represent the GR and $1 / \mathrm{f}$ noise sources generated in the $R_{B}$ and $R_{E}$ resistances.

$\delta \mathrm{I}_{\mathrm{BC}}$ represents the $\mathrm{GR}$ and $1 / \mathrm{f}$ noise sources generated into the base-collector junction

$\delta \mathrm{I}_{\mathrm{RBC}}$ represents the GR and $1 / \mathrm{f}$ noise sources of the recombination current of the base-collector junction.

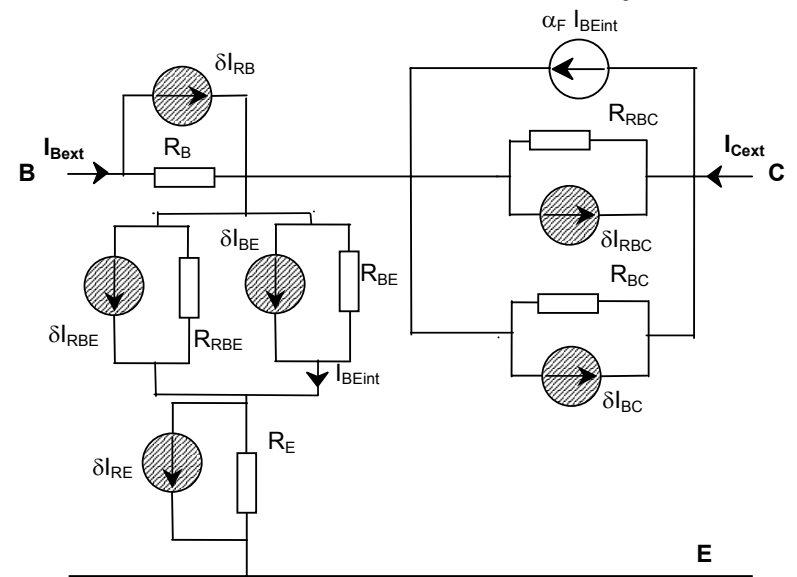

Fig. $\overline{\text { 1: HBT small signal differential model with possible localization of }}$ the low frequency noise current sources 


\section{B. EXTRACTION FROM MEASUREMENTS OF THE LOW FREQUENCY NOISE MODEL}

1. Determination of the HBT low frequency noise model

The principle of the low frequency measurement set-up is shown Fig. 2. This new set-up, developed at IRCOM Institute, is an improved version of the one described elsewhere.

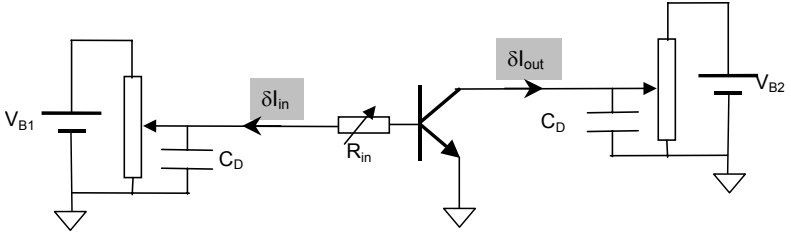

Fig.2: basic noise measurement set-up

First measurements allow to eliminate some of the possible noise sources as main noise sources. Further measures allow to locate the two dominant low frequency noise sources

2. Accurate extraction of the two main low frequency noise sources

Fig. 3 represents the low frequency HBT model retained.

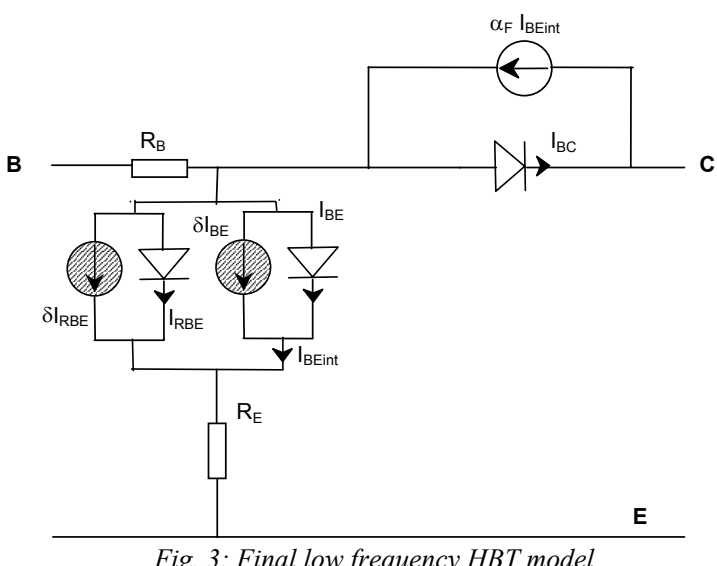

The spectral densities of $\delta I_{R B E}$ and $\delta I_{B E}$ are respectively function of the current flowing in the diodes $\mathrm{I}_{\mathrm{RBE}}$ and $\mathrm{I}_{\mathrm{BE}}$.

\section{Scalable noise model.}

For the determination of the scalable model, the unit length is the micrometer and the unit current the Ampere. All the measured transistors of this study having the same width $\mathrm{W}=2 \mu \mathrm{m}$. For the noise sources of surface G$\mathrm{R}$ [3], we can write:

$$
S_{\delta I_{R B E}}=\frac{K_{R B E}}{L^{m} N^{k}} J_{b e 0}^{p}
$$

Where the section area writes as : $\quad \mathrm{A}=\mathrm{N} \mathrm{W} \mathrm{L}$

$$
\begin{array}{r}
\text { N: finger number } \\
\text { W: emitter width } \\
\text { L: emitter length } \\
J_{b e O} \text { :current density }
\end{array}
$$

Equation (1) is fitted by taking into account all the measured transistors from $1 \times 2 \times 20$ to $6 \times 2 \times 40 \mu \mathrm{m}^{2}$ size. For the 10 measured transistors, $10 \log \left(S_{\delta I_{R B E}} L^{m} N^{k}\right)$ is plotted versus $\log \left(J_{b e 0}\right)$.

Fig. 4 show the results for a frequency of $1 \mathrm{kHz}$.

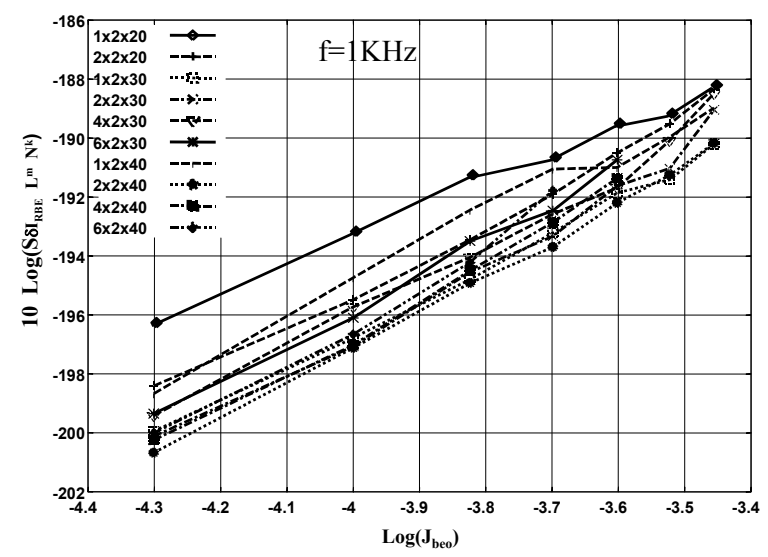

Fig. $410 \log \left(S_{\delta I_{R B E}} L^{m} N^{k}\right)$ computed from (1) for $f=1 \mathrm{KHz}$

A very good fit is obtained in the full range of bias current density, from $0,05 \mathrm{~mA} / \mu \mathrm{m}^{2}$ to $0,3 \mathrm{~mA} / \mu \mathrm{m}^{2}$ for all the transistors but the plot relative to the transistor of one finger $2 \times 20 \mu \mathrm{m}^{2}$ size deviates from the others. This problem is often encountered with transistors of small size whose actual dimensions must be precisely known in order to obtain accurate scalability.

For the volume G-R noise sources, a realistic expression must take into account many different traps, nonhomogeneously distributed. A generalized expression will take the form:

$$
S_{\delta I_{B E}}=A^{v} K_{B E} J_{b e 0}^{r}
$$

This expression constitutes an accurate model of the volume L.F. noise of the emitter-base junction, for the HBT process.

For the 10 measured transistors, $10 \log \left(S_{\delta I_{B E}} / A^{v}\right)$ is plotted versus $\log \left(\mathrm{J}_{\mathrm{be} 0}\right)$. Fig. 5 shows the results for a frequency of $1 \mathrm{kHz}$.

We note an excellent agreement of the scalable model of the low frequency noise source $S_{\delta I_{B E}}$.

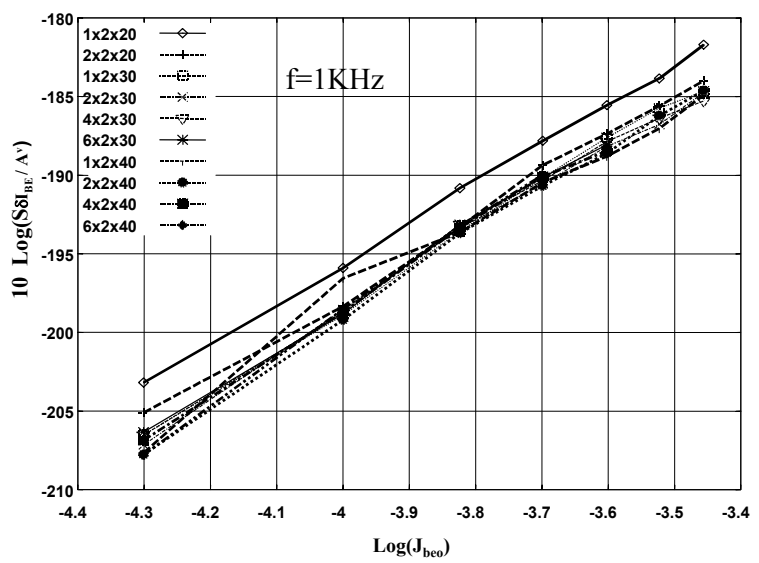

Fig. 5: $10 \log \left(S_{\delta I_{B E}} / A^{v}\right)$ computed from (2) for $f=1 \mathrm{kHz}$ 
-Resulting cyclostationary Norton noise current sources -In dynamic operating conditions, the shot noise source, which is a white noise source, is fully modulated ; it results a cyclostationary spectral density $S_{\delta I}$ shot proportional to the dynamic current density $J_{b e}$. -For the low frequency noise sources, the modulation theory is still under development [4-7]. So, physicsbased empirical expressions will be used. It may be inferred with an accurate assumption, that the modulation function of the spectral density $S_{\delta I_{B E}}$ and $S_{\delta I_{R B E}}$ can be taken as proportional to the square of the dynamic current density $J_{b e}$. These modulation functions have been used for the design of the VCOs. Phase noise results obtained on the processed oscillators clearly justify the proposed empirical LF noise model.

\section{IMPLEMENTATION OF COMPLETE NOISE $M O D E L$}

In order to include the resulting noise current sources in the transistor $\pi$-model of the UMS HBT process, some straightforward transformations from $\mathrm{T}$ to $\pi$-model have been performed. For the sake of brevity they are not detailed here.

\section{FULLY INTEGRATED LOW NOISE VCO}

\section{A. CIRCUIT DESCRIPTION AND SIMULATION}

\section{Circuit principle}

A push-push oscillator (figure 6) consists of two symmetric oscillator parts, which are operating at half the desired frequency $\left(\mathrm{f}_{0}=\mathrm{f}_{\text {OUT }} / 2\right)$, driving a phase difference of $180^{\circ}$. The circuit can be considered as two singleended symmetrical oscillators connected in parallel to a virtual ground. In the proper operation of a Push-Push oscillator, power is delivered to the load only at the second harmonic frequency, but some complementary output can be considered at fundamental frequency. Therefore, absolute symmetry of the two sub-circuits and output network is required to achieve an efficient operation of the circuit.

This design approach was adopted to decrease the $1 / \mathrm{f}$ noise generation and its transposition around the carrier.

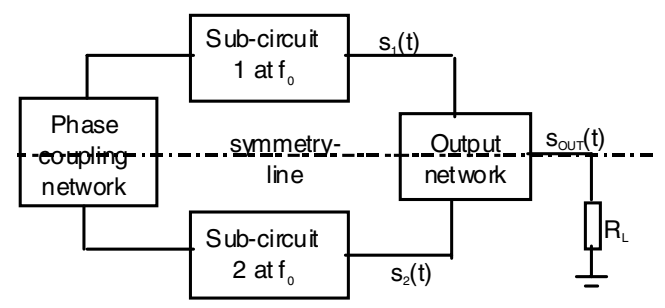

Figure 6: Push-push oscillator concept

\section{Oscillatot design}

The MMIC VCO is fabricated using a $2 \mu \mathrm{m} \mathrm{InGaP/GaAs}$ HBT technology which provides $\mathrm{f}_{\mathrm{T}}$ 's and $\mathrm{f}_{\max }$ 's of 50 $\mathrm{GHz}$ and $70 \mathrm{GHz}$ respectively.

The design of a push-push oscillator starts with the single ended oscillator and in particular with the active device. The ADS simulator was used as CAD tools. The active part can be considered as an one port device [8]. One of the tree terminals of the transistor, in our case the base, is terminated with a resonator which is defined by a $Q$ factor, load impedance and a resonance frequency. The transistor (size and biasing point) and the feedback capacitance $\mathrm{C}_{\mathrm{ce}}$ (figure 7 ) are optimised for having enough negative resistance for compensating the resonator loss. The resonator design mainly consists of $\mathrm{Q}$ factor optimisation. This part begins with a linear analysis to determine the condition of oscillation and continue with a non-linear analysis to estimate the main characteristics of the VCO (output power, centre frequency, bandwidth, phase noise...). During this phase a trade-off is done between all the contributions from all the noise sources.

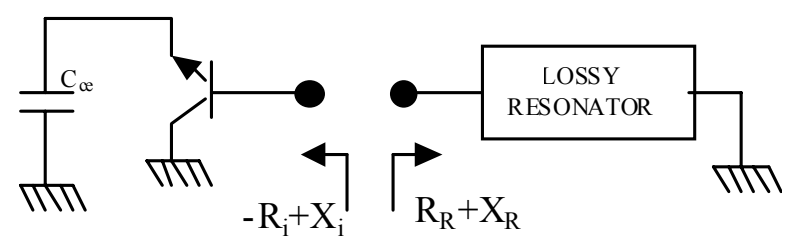

Figure 7: Basic oscillator circuit description The next step consists of joining both sub-circuits to achieve the push-push VCO. This part allows to determine the final characteristics of the circuit. The simulations shows a centre frequency of $2 \mathrm{GHz}$ with a bandwidth of $300 \mathrm{MHz}$ (figure 8) and a phase noise of $95 \mathrm{dBc} / \mathrm{Hz}$ at $10 \mathrm{kHz}$ from carrier at $2 \mathrm{GHz}$.

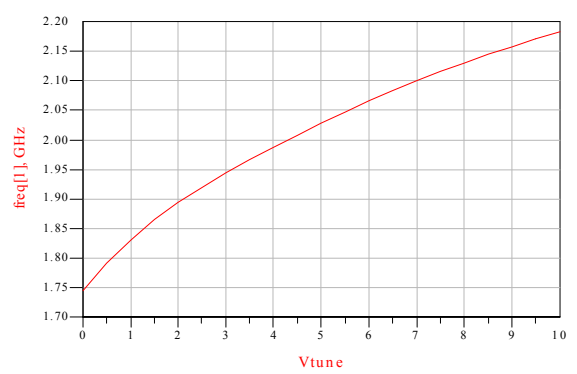

Figure 8: Simulated frequency tuning characteristic versus tuning voltage

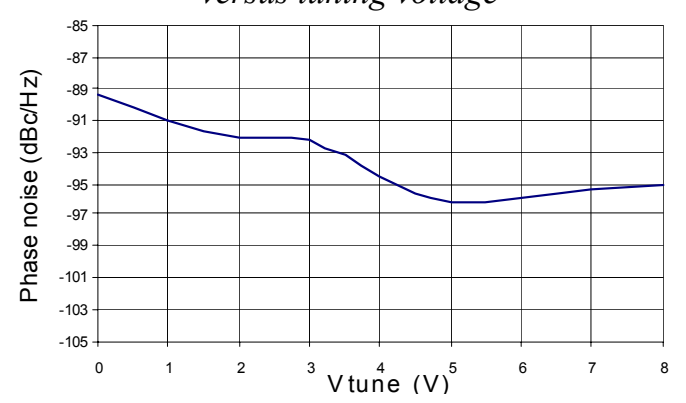

Figure 9: Simulated phase noise at $10 \mathrm{KHz}$ offset from carrier versus tuning voltage 


\section{B. MEASURED PERFORMANCE}

The VCO has been characterized on-wafer for chip selection and then in test fixture for full characterization. The frequency response is given in figure 10, the centre frequency is $2 \mathrm{GHz}$, the tuning range is around 280 MHz.

Phase noise measurements have been done on a millimetre wave test bench based on frequency transposition and on delay line at IF frequency. The phase noise spectrum versus frequency offset from the carrier is shown on figure 11 . At $2 \mathrm{GHz}$, the phase noise is $-95 \mathrm{dBc} / \mathrm{Hz}$ at $10 \mathrm{kHz}$ from carrier, and $-118 \mathrm{dBc} / \mathrm{Hz}$ at $100 \mathrm{kHz}$. Moreover, the figure 12 shows the phase noise increase for some low tuning voltages. This results are in good accordance with the predicted simulation results.

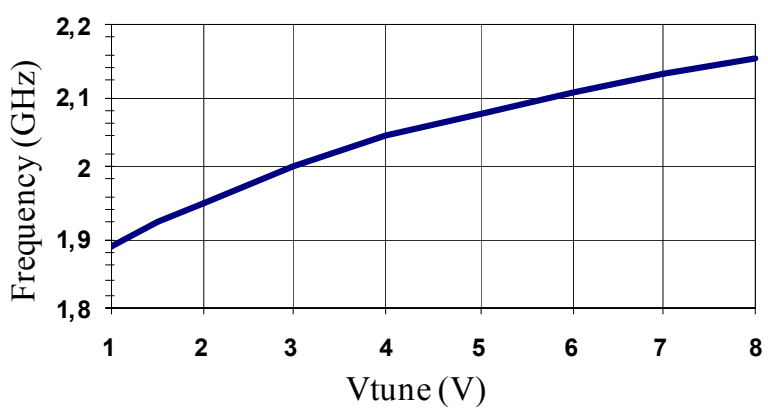

Figure 10: Frequency tuning characteristic versus tuning voltage

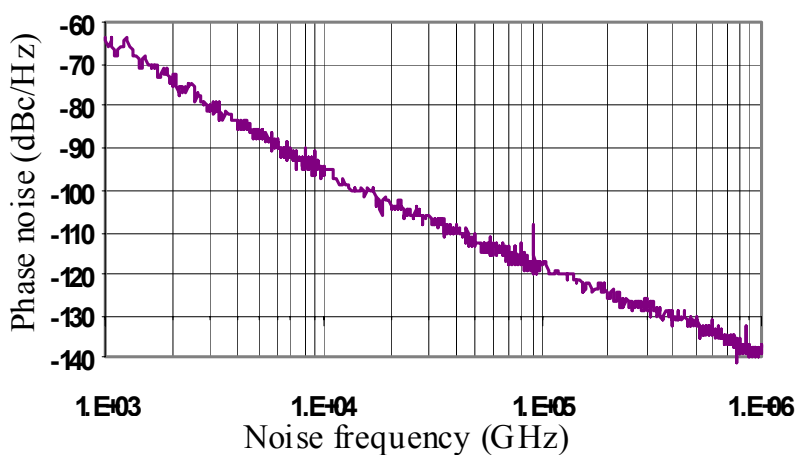

Figure 11: Measured phase noise for a tuning voltage at $4 V$ versus frequency offset from carrier

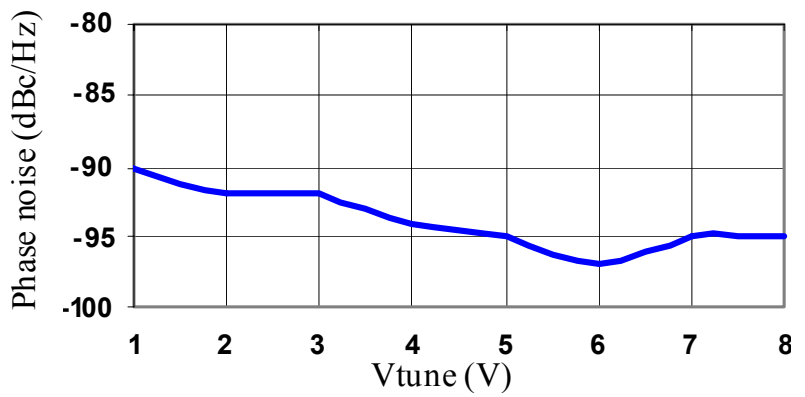

Figure 12: Measured phase noise at $10 \mathrm{KHz}$ offset from carrier versus tuning voltage

\section{CONCLUSION}

The realisation of a fully integrated S band VCO using UMS HBT process has been described. It exhibits at $2 \mathrm{GHz}$ a phase noise of $-95 \mathrm{dBc} / \mathrm{Hz}$ at $10 \mathrm{KHz}$ from carrier and a tuning range of $280 \mathrm{MHz}$. These performances have been obtained thanks to the advanced noise model of the HBT transistor for the optimisation of the phase noise level and a specific work on design robustness versus technology spreads.

\section{ACKNOWLEDGEMENT}

The authors would like to thank SIAE for their continous and active support.

\section{REFERENCES}

[1] A. Pénarier, F. Pascal, et al "Low-frequency noise of InP/InGaAS Heterojunction Bipolar Transistors", Jpn. J. Appl. Phys., 2001, 40, pp 525-529.

[2] R.Plana, J.P Roux, et al. "Excess noise in microwave $\mathrm{GaInP} / \mathrm{GaAs}$ Heterojunction bipolar transistors "Proceedings of European GaAs and related III-V compounds applications symposium, Torino, Italy, April 1994,pp131-134.

[3] O. Jantsch, " Flicker (1/f) noise generated by random walk of electrons in interfaces", IEEE Transations on Electron Devices, Vol. ED 34, May1987, pp 1100-1115

[4] S. Perez, S.L. Delage, et al. "Microscopic analysis of generation-recombination noise in semiconductors under dc and time varying electric fields", Journal of applied Physics, vol 88, $\mathrm{n}^{\circ} 2,15$ July 2000

[5] A. Cappy, F. Danneville, et al. "Noise analysis in devices under nonlinear operation", Solid-state electronics, vol43, 1999, pp 21-26

[6] J.E. Sanchez, G. Bosman and M.E. Law "TwoDimensional Semiconductor Device Simulation of Trap-Assisted Generation-Recombination Noise Under Periodic Large-Signal Conditions and Its Use for Developing Cyclostationary Circuit Simulation Models." IEEE Transations on Electron Devices, Vol. ED 50, №5, May 2003, pp 1353-1362.

[7] F.Bonani, S. Donati Guerrieri, G. Ghione, "Noise source modeling for cyclostationary noise analysis in large-signal device operation", IEEE Trans. On Electron Devices, Vol. 49, Issue 9, sept.2002, pp 1640-164.

[8] J.W. Boyles, "The Oscillator as a Reflection Amplifier. An Intuitive Approach to Oscillator Design", Microwave Journal, June 1986, pp 83-98. 